\title{
Shingles in palmar cutaneous branch of ulnar nerve
}

\author{
Hong Ki Min ${ }^{1}$, Se Hee Kim ${ }^{1}$, Sang-Heon Lee ${ }^{2}$, and Hae-Rim Kim²
}

${ }^{1}$ Division of Rheumatology, Department of Internal Medicine, Konkuk University Medical Center, Seoul; ${ }^{2}$ Division of Rheumatology, Department of Internal Medicine, Konkuk University Medical Center, Konkuk University School of Medicine, Seoul, Korea

Received: March 4, 2020

Revised : March 25, 2020

Accepted: March 26, 2020

\section{Correspondence to}

Hae-Rim Kim, M.D.

Tel: $+82-2-2030-7542$

Fax: +82-2-2030-7728

E-mail: kimhaerim@kuh.ac.kr https://orcid.org/0000-0002$1911-6236$
Shingles on the wrists or hands is rare and few reports in the available literature have described such patients. A 76-year-old woman with known rheumatoid arthritis (RA) visited the outpatient clinic with a 3-day history of left wrist pain. She was diagnosed with RA 4 years prior to presentation and presently received disease-modifying antirheumatic drugs (methotrexate and tacrolimus). Physical examination showed vesicles and skin rash localized to the ulnar aspect of her left palm along the area of innervation of the palmar cutaneous branch of the ulnar nerve (Fig. 1A). The erythrocyte sedimentation rate and serum high-sensitivity C-reactive protein level were within the reference range. Shingles rarely affects the palm; however, the clinical presen- tation in this patient was compatible with shingles. Therefore, we initiated famciclovir and pregabalin therapy. The vesicles and skin rash improved following a 1-week course of antiviral treatment (Fig. 1B); however, neuropathic pain persisted. Pregabalin was administered for 4 additional weeks for post-herpetic neuralgia. Five weeks after the initial symptoms, the patient's neuralgia disappeared with only a few residual lesions observed on the palm (Fig. 1C). Shingles occurs secondary to reactivation of the varicella zoster virus and usually presents as a band-like distribution of vesicles along the affected dermatome. The varicella zoster virus persists in the sensory nerve ganglion after primary infection, with the most common sites of involvement being
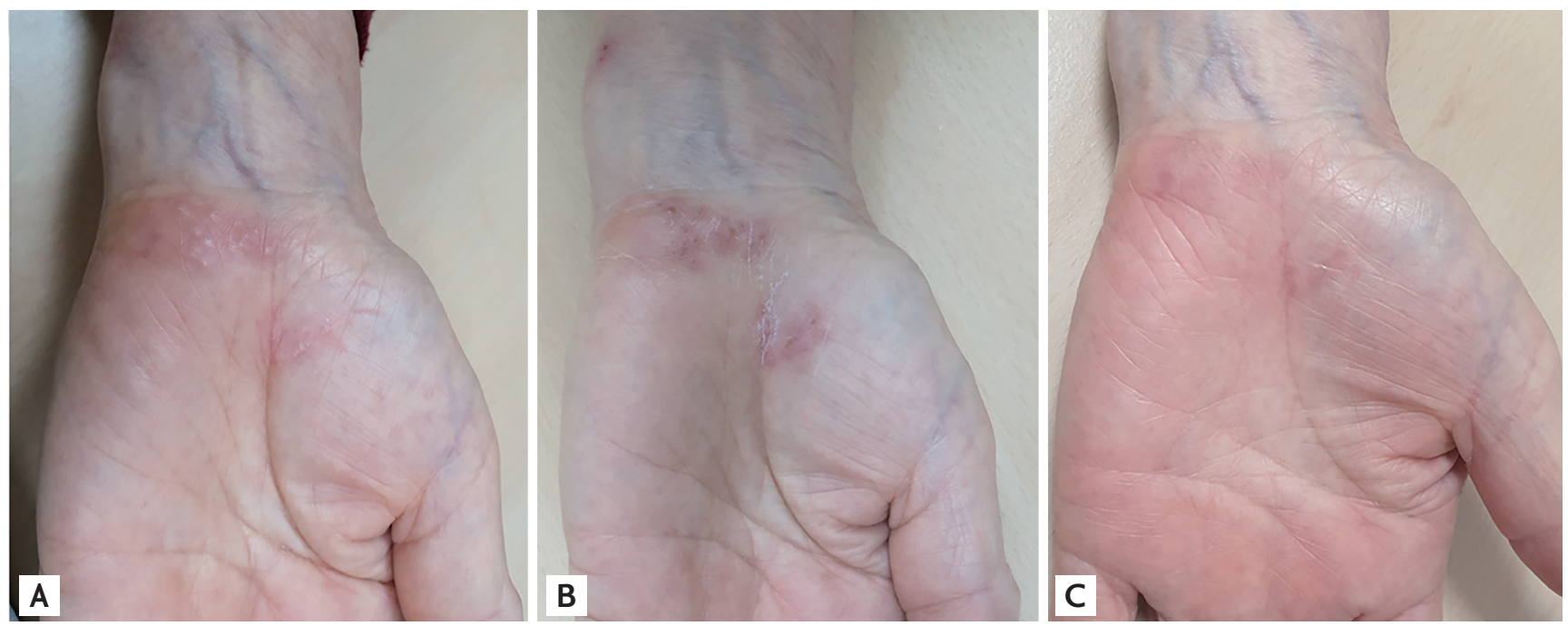

Figure 1. (A) Photograph obtained at the patient's initial clinical visit showing multiple bullous vesicles and skin rash on the palmar aspect of the wrist. (B) Photograph obtained 1 week after famciclovir treatment showing improvement in vesicles and skin rash. (C) Photograph obtained 5 weeks after the patient's initial presentation showing only residual lesion 
the thoracic dermatomes and trigeminal nerve. The sensory branch of the ulnar nerve divides into three branches (the palmar cutaneous, dorsal cutaneous, and the superficial sensory branch); the palmar cutaneous branch innervates the hypothenar eminence. This case report highlights a rare presentation of shingles localized to the palmar cutaneous branch of the ulnar nerve. Clinicians should perform a thorough physical examination to distinguish shingles-induced pain from that associated with arthritis aggravation in patients with RA. Informed consent was obtained from the patient for publication of this case report and accompanying images.

\section{Conflict of interest}

No potential conflict of interest relevant to this article was reported. 